\title{
Cooperative Routing for Distributed Detection in Large Sensor Networks
}

\author{
Youngchul Sung, Saswat Misra, Lang Tong, and Anthony Ephremides
}

\begin{abstract}
In this paper, the detection of a correlated Gaussian field using a large multi-hop sensor network is investigated. A cooperative routing strategy is proposed by introducing a new link metric that characterizes the detection error exponent. Derived from the Chernoff information and Schweppe's likelihood recursion, this link metric captures the contribution of a given link to the decay rate of error probability and has the form of the capacity of a Gaussian channel with the sender transmitting the innovation of its measurement. For one-dimensional GaussMarkov fields, the link metric can be represented explicitly as a function of the link length. Cooperative routing is achieved using the Kalman data aggregation and shortest path routing. Numerical simulations show that cooperative routing can be significantly more energy efficient than noncooperative routing for the same detection performance.
\end{abstract}

Index Terms-Chernoff information, Cross-layer design, Distributed detection, Innovations process, Network routing

\section{INTRODUCTION}

W IRELESS sensor networks are often deployed to perform a specific set of tasks. Therefore, the design of such a network should be optimized for these tasks. Consider, for example, a network of distributed sensors for the detection of certain events or phenomena. Sensors may have a limited communications range, and their local detection may be unreliable. Thus, it is necessary to aggregate the data collected from multiple sensors and send them to a fusion center where a global decision can be made. The measurements at individual sensors, however, are often spatially correlated, and delivering all of this raw data to the fusion center may be both inefficient (in utilization of the network's resources) and unnecessary (for accurate detection). It is in this context that cooperative networking is especially appealing for sensor networks.

We consider the problem of cooperative routing for distributed detection of a correlated random signal field. We assume that the network covers a large geographical area and

Manuscript received February 1, 2006; revised July 1, 2006. This work was supported in part by the U. S. Army Research Laboratory under the Collaborative Technology Alliance Program, Cooperative Agreement DAAD19-012-0011 and the National Science Foundation under Contract CNS-0435190. The U. S. Government is authorized to reproduce and distribute reprints for Government purposes notwithstanding any copyright notation thereon. Part of this work was presented as a plenary at the 2005 IEEE International Conference on Acoustics, Speech, and Signal Processing (ICASSP), Philadelphia, PA, USA.

Y. Sung is with Qualcomm Inc., San Diego, CA 92121, USA (e-mail: ysung@qualcomm.com).

S. Misra is with the Army Research Laboratory, Adelphi, MD 20783, USA (e-mail: smisra@arl.army.mil).

L. Tong is with the School of Electrical and Computer Engineering, Cornell University, Ithaca, NY 14853, USA ( e-mail 1tong@ece.cornell.edu).

A. Ephremides is with Dept. of Electrical Engineering, University of Maryland, College Park, MD 20742, USA (e-mail: tony@eng.umd.edu).

Digital Object Identifier 10.1109/JSAC.2007.070221.

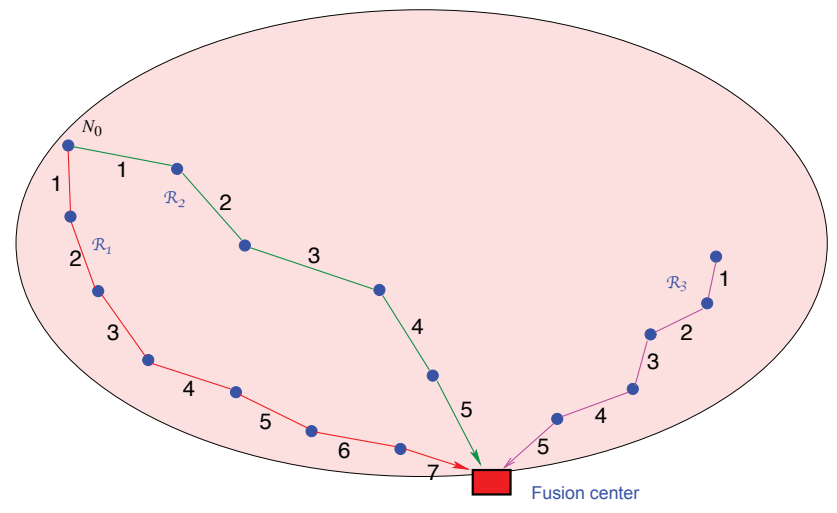

Fig. 1. Routing for detection in a multi-hop wireless sensor network.

that sensor measurements need to be sent to a fusion center through a multi-hop network as illustrated in Fig. 1. Two modes of operation are commonly of interest. In clock-driven applications, the fusion center initiates data collection in some target area of the network or through random sampling of the sensor field. In event-driven applications, on the other hand, certain sensors are alarmed locally and send their data to the fusion center. In both cases, additional information is collected by the intermediary sensors along the chosen route, and the problem is to choose an appropriate route that gives the best detection performance under operating constraints.

Some illustrative scenarios will help to highlight two key components of cooperative routing: (i) the need of a link metric that connects detection performance with energy consumption, and (ii) an optimal data aggregation scheme that facilitates cooperation.

Suppose first that the fusion center decides to probe the network, and that it has chosen a set of nodes, say those on route $\mathcal{R}_{3}$ in Fig. 1, to gather data (an optimized algorithm to select such a route is part of the problem addressed in this paper). A noncooperative scheme would treat data along $\mathcal{R}_{3}$ as independently generated, and every sensor would deliver its local data to the fusion center. The number of messages transmitted grows roughly in the order $O\left(n^{2}\right)$ where $n$ is the number of nodes on the route. Can cooperative routing reduce the required number of transmissions while maintaining the same detection performance? This answer depends on our ability to combine data measured at different locations and, of course, the correlation structure of the measurements. To this end, the role of optimal data aggregation is crucial.

Suppose next that sensor $N_{0}$ in Fig. 1 is to initiate a report to the fusion center, and that it compares two different routes, $\mathcal{R}_{1}$ versus $\mathcal{R}_{2}$. Route $\mathcal{R}_{1}$ has more hops, and thus it gathers 
measurements from more sensors. But it is hardly obvious that $\mathcal{R}_{1}$ leads to better detection performance. Nodes on $\mathcal{R}_{1}$ are more closely spaced, and presumably take more correlated measurements than those on $\mathcal{R}_{2}$. If sensor measurements have strong spatial correlation, it is possible that data on $\mathcal{R}_{1}$ is less informative. Furthermore, having more nodes on the route means more transmissions, which leads to higher energy consumption. The tradeoff between performance and energy makes route selection nontrivial. What we need is an analytical characterization of performance and energy consumption, one that leads to a link metric on which route selection is based.

\section{A. Summary of Results and Organization}

This paper focuses on two aspects of cooperative routing: the definition of a link metric for cooperation and a method of cooperative data aggregation. To gain insights into the tradeoffs between performance and energy consumption, we consider the problem of detecting a correlated Gaussian field in Gaussian noise. Special attention is paid to the onedimensional Gauss-Markov field for which the Markovian property makes optimal data aggregation simpler and more efficient. We note that the Gaussian field assumption and the Gauss-Markov correlation model are related to the practical problem of detecting diffusive gas modeled as an OrnsteinUhlenbeck process [1].

In Section III, we propose a new link metric using the Chernoff information which allows us to characterize detection performance based on the number and the locations of sensors along the route. Intuitively, Chernoff information measures the efficiency with which data collection reduces error probability, i.e., the higher the Chernoff information, the faster the error probability decays as the number of measurements increases. The Chernoff information, however, does not directly suggest an implementation of optimal routing. Using Schweppe's recursive representation of the likelihood function [2], we show that, for medium and high Signal-to-Noise Ratio (SNR), the link metric that leads to the maximum Chernoff information corresponds to the capacity of a Gaussian channel where the sender transmits the innovation of its measurement to the receiver. Thus, the proposed link metric suggests that it is the innovations, not the raw data, that should be propagated to the fusion center.

In Section IV, we show how the proposed link metric leads to a strategy for optimal data aggregation through cooperative transmission. Specifically, we consider the special case when the signal correlation is described by a onedimensional Gauss-Markov process and show that optimal data aggregation can be performed via a Kalman filter. The proposed optimal aggregation scheme reduces the amount of information exchange required at each node, and consumes the same amount of energy for each node in the route.

In Section V, we use these results to propose an optimized routing strategy. Since choosing a route to maximize Chernoff information without any constraints leads to one that traverses the entire network, we introduce energy consumption into the routing problem. Because both the link metric and the transmission energy are functions of the length of the link, we are able to cast the routing problem as one of maximizing accumulative link metrics subject to energy constraints. We propose an efficient shortest path routing [3] implementation of our new link metric and evaluate performance.

\section{B. Related Work}

The practical problem considered in this paper is one of distributed detection in which sensors collect spatially correlated information and deliver their data to a fusion center for optimal detection. See [4]-[6] and references therein for classical results. For large wireless sensor networks, issues of imperfect transmission [7], [8], medium access control [9], [11]-[13], and energy constraints [14], [15] have attracted attention recently. The use of asymptotic techniques to characterize performance for distributed detection has been presented in the literature [10], [11], [13], [14], [16]; most assume conditionally independent observations.

Routing, to our knowledge, has not been considered for distributed detection. This is partly because the assumption of conditional independence of observations trivializes the issues of where to collect data and optimal data aggregation. Once the independence assumption is removed, data collected along different routes may result in different detection performance. We note, however, that our focus is different from that of classical distributed detection. We do not treat the problem of local quantization at sensor level. We assume that sensors deliver unquantized measurements to the fusion center, and focus on the issue of where to collect data and how to deliver data to the fusion center in an energy efficient fashion. The recent results of Sung, Tong, and Poor [17] characterizing the error exponent for the detection of Gauss-Markov processes are related to this paper. The contribution here beyond [17] is the derivation of a link metric for multi-hop sensor networks. The theme of cross-layer design to incorporate signal processing performance measures into routing is outlined in [18].

The literature on routing in wireless ad hoc networks is vast. We focus on works which have similar design considerations as our own, i.e., the definition of link metric and/or distributed data aggregation. A common link metric for ad hoc networks is the number of hops. Examples include the destination sequenced distance vector (DSDV) [19], the dynamic source routing (DSR) [20], and the temporally ordered routing algorithm (TORA) [21]. To incorporate application performance or data quality into routing in sensor network, many protocols have been proposed. Examples include query-based datacentric protocols [22], [23] and variants designed to increase the energy efficiency and reliability of the network [24], [25]. Examples of cross-layer approaches combining routing with performance in various layers can be found in [26]-[30]. In [30], the authors investigated the effect of spatial correlation between signal sources on routing and data compression via the total energy consumption in the network to transfer overall information to the gateway node. Energy-based routing has been studied from various perspectives. For example, the battery power of nodes and the necessary transmission power between neighboring nodes are considered in [31]-[34].

Methods of data aggregation in sensor networks include Directed Diffusion [22], Sensor Protocols for Information via 
Negotiation (SPIN) [35], Tiny AGreggation (TAG) [36], eScan [37], and Power Efficient GAthering in Sensor Information Systems (PEGASIS) [38]. In the latter, data is aggregated and fused by each sensor en route in order to increase the signal-to-noise ratio of the desired data. However, the routing and aggregation mechanisms are not explicitly related to application performance, as they are not designed for a particular application. In [39], the aggregation-induced tradeoff between energy consumption and the delay of information propagation is studied as a function of source-sink placements and the density of the network. The effect of timing in data aggregation algorithms was investigated in [40]. In addition, several works have studied the use of a Kalman filter (or its variants) to exploit the correlation of sensor readings for improved data aggregation while minimizing communications costs, e.g., see [41], [42] and the references therein.

\section{SySTEM MOdeL}

We specify in this section the sensor network and formulate the detection process under the Bayesian framework. The notations are standard: random variables are denoted by capital letters (e.g., $Y$ ) and their realizations by the corresponding lower case $(y)$. The expectation operator is denoted by $\mathbb{E}(\cdot)$, and $\mathbb{E}_{j}(\cdot)$ denotes the expectation conditioned on an event $\mathcal{H}_{j}$. The notation $\mathbf{X} \sim \mathcal{N}(\boldsymbol{\mu}, \boldsymbol{\Sigma})$ means that $\mathbf{X}$ is a real-valued Gaussian random vector with mean $\boldsymbol{\mu}$ and covariance matrix $\boldsymbol{\Sigma}$. Finally, for a matrix $\mathbf{A}, \mathbf{A}(i, j)$ is the $(i, j)$ entry of $\mathbf{A}$.

\section{A. Sensors, Measurement Model, and Detection at the Fusion Center}

Consider a sensor network where each sensor knows its own location. Sensors have a fixed transmission range, which implies a connectivity graph, and necessitates multi-hop routing. Suppose, without loss of generality, that the detection process originates at a node $N_{0}$ and that the fusion route traverses nodes $N_{0}, N_{1}, \cdots, N_{n-1}$ where $N_{n-1}$ (also denoted $F$ ) is the fusion center. Let $\mathcal{R}\left(N_{0}, \cdots, N_{n-1}\right)$ denote this route and $\mathbf{x}_{i}$ a two-dimensional vector containing the location of the $i$ th sensor along this route. Let $\Delta_{i} \triangleq\left\|\mathbf{x}_{i}-\mathbf{x}_{i-1}\right\|$ denote the Euclidean distance between sensors $N_{i}$ and $N_{i-1}$. The only structure we impose on a route is that each node be visited no more than once. For example, Figure 2 depicts two possible routes $\mathcal{R}\left(N_{0}, \cdots, N_{n-1}\right)$ from $N_{0}$ to the fusion center for a given topology.

Let hypothesis $\mathcal{H}_{1}$ denote the presence of the phenomenon within the sensor network and $\mathcal{H}_{0}$ its absence. The observations along route $\mathcal{R}$ under each hypothesis are given by

$$
\begin{aligned}
& \mathcal{H}_{0}: Y_{i}=W_{i}, \quad i=0,1, \cdots, n-1, \\
& \mathcal{H}_{1}: Y_{i}=S_{i}+W_{i}, \quad i=0,1, \cdots, n-1,
\end{aligned}
$$

where $Y_{i}$ is the observation at sensor $i$, and where the $S_{i}$ 's are correlated Gaussian samples of the signal with $S_{i} \sim$ $\mathcal{N}\left(0, \sigma_{S}^{2}\right)$ and covariance matrix $\boldsymbol{\Sigma}_{S}(i, j)=\mathbb{E}_{1}\left(S_{i} S_{j}\right)$. The noise samples $W_{i}$ are i.i.d. Gaussian with $W_{i} \sim \mathcal{N}\left(0, \sigma_{W}^{2}\right)$. The SNR of sensor measurements is

$$
\mathrm{SNR}=\frac{\sigma_{S}^{2}}{\sigma_{W}^{2}} .
$$

The fusion center makes the detection based on collected observations $\left\{Y_{i}=y_{i}\right\}$. The detector which minimizes the probability of detection error for (1) is given by the likelihood ratio detector

$\delta_{B}\left(y_{0}, \cdots, y_{n-1}\right)= \begin{cases}\mathcal{H}_{1}, & \ln \frac{p_{1}\left(y_{0}, \cdots, y_{n-1}\right)}{p_{0}\left(y_{0}, \cdots, y_{n-1}\right)} \geq \tau \triangleq \ln \frac{\pi_{0}}{\pi_{1}}, \\ \mathcal{H}_{0}, & \text { otherwise, }\end{cases}$

where $p_{j}\left(y_{0}, \cdots, y_{n-1}\right), j=0,1$, is the probability density function of the jointly Gaussian random variables under $\mathcal{H}_{j}$, and where $\pi_{j}$ is the prior probability of $\mathcal{H}_{j}$.

\section{B. Energy Consumption}

It is necessary to specify an energy consumption model. We assume that all real numbers processed by sensors (observations and auxiliary computation variables) are quantized with sufficiently high precision to ignore quantization error. We assume that the energy consumed in routing a (quantized) real number from sensor $N_{i-1}$ to sensor $N_{i}$ is

$$
E_{i}=E_{P}+E_{T} \Delta_{i}^{\nu},
$$

where $E_{P}$ is the processing energy and $E_{T} \Delta_{i}^{\nu}$ the transmission energy, which is a function of the link length $\Delta_{i}$ according to a power-law. We assume that the attenuation coefficient $\nu \geq 1$.

As examples, note that a naive noncooperative routing where measurements from all sensors on a route $\mathcal{R}\left(N_{0}, \cdots, N_{n-1}\right)$ are delivered individually to the fusion center consumes a total energy

$$
E_{\mathcal{R}}^{\mathrm{nc}}=\sum_{i=1}^{n-1} \sum_{j=i}^{n-1}\left(E_{P}+E_{T} \Delta_{j}^{\nu}\right)
$$

which grows in the order of $O\left(n^{2}\right)$, whereas the proposed cooperative routing strategy with Kalman aggregation (to be presented in Section IV-B) will be seen to consume a total energy

$$
E_{\mathcal{R}}^{\mathrm{c}}=\sum_{i=0}^{n-1} \alpha E_{P}+\beta E_{T} \Delta_{j}^{\nu}
$$

where $\alpha \geq 0$ and $\beta \geq 0$ are constants independent of $n$ and sensor locations that describe the relative processing and transmission energy per observation needed by cooperative routing relative to non-cooperative routing. Note that $E_{\mathcal{R}}^{\mathrm{c}}$ grows only in the order $O(n)$.

\section{Link Metric FOR COOPERATIVE Routing}

In this section we derive a new link metric for the detection of widespread phenomena in a large scale ad-hoc wireless sensor network. First, we propose using Chernoff information as a tractable metric which captures the probability of detection error of a given route. Next, we use Schwappe's recursive representation of the likelihood function to express the Chernoff information in a desirable form. From this, we extract a link metric, seen to correspond to the capacity of the Gaussian channel with the signal power given by the power of the innovations process, which characterizes the contribution of each link to detection performance. 


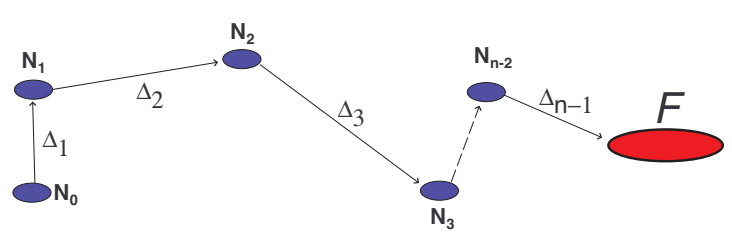

(a)

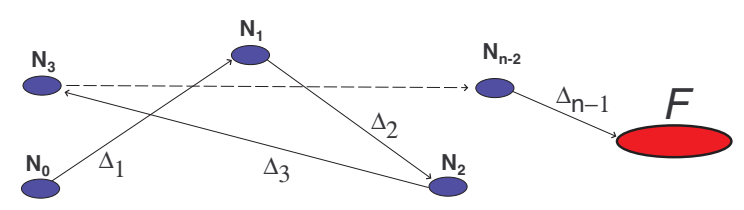

(b)

Fig. 2. Two examples of fusion routes $\mathcal{R}\left(N_{0}, \cdots, N_{n-1}\right)$.

\section{A. Chernoff Information and Schweppe's Recursion}

Consider a fusion route $\mathcal{R}\left(N_{0}, \cdots, N_{n-1}\right)$ and define the vector of observations along the route:

$$
\mathbf{Y}_{n} \triangleq\left[Y_{0}, \cdots, Y_{n-1}\right]^{T} \text {. }
$$

The distribution of $\mathbf{Y}_{n}$ is given by

$$
\mathbf{Y}_{n} \sim \begin{cases}\mathcal{N}\left(\mathbf{0}, \sigma_{W}^{2} \mathbf{I}\right) & \text { under } \mathcal{H}_{0} \\ \mathcal{N}\left(\mathbf{0}, \boldsymbol{\Sigma}_{S}+\sigma_{W}^{2} \mathbf{I}\right) & \text { under } \mathcal{H}_{1}\end{cases}
$$

where $\mathbf{I}$ is the $n \times n$ identity matrix. The probability of detection error is $P_{\mathcal{E}}=\pi_{0} P_{\mathcal{E} \mid \mathcal{H}_{0}}+\pi_{1} P_{\mathcal{E} \mid \mathcal{H}_{1}}$, where $P_{\mathcal{E} \mid \mathcal{H}_{j}}$ is probability of detection error conditioned on hypothesis $\mathcal{H}_{j}$. The Chernoff bound on $P_{\mathcal{E}}$ is [43]

$$
P_{\mathcal{E}} \leq \pi_{0}^{1-s} \pi_{1}^{s} e^{\mu(s)}, 0 \leq s \leq 1,
$$

where $\mu(s)$ is the cumulant generating function of the loglikelihood ratio under $\mathcal{H}_{0}$, i.e.,

$$
\mu(s)=\ln \mathbb{E}_{0}\left\{e^{s \ln \frac{p_{1}\left(Y_{0}, \cdots, Y_{n-1}\right)}{p_{0}\left(Y_{0}, \cdots, Y_{n-1}\right)}}\right\}, \quad 0 \leq s \leq 1 .
$$

The Chernoff information [44] is defined as the cumulant generating function evaluated at the maximizing value of $s$, i.e.,

$$
C\left(p_{0}, p_{1}\right) \triangleq \sup _{0 \leq s \leq 1}\{-\mu(s)\},
$$

which is nonnegative.

The conventional procedure for the calculation of Chernoff information involves a quadratic form of the observation vector (2) and the eigenvalues of $\boldsymbol{\Sigma}_{S}$ [43, pp.89-90]. A mathematically equivalent approach that provides insights into the contribution of each link to $P_{\mathcal{E}}$ of a route can be obtained from the Schweppe's recursive representation of the likelihood function [2], which we describe next.

Decompose the log-likelihood as follows:

$\ln p_{1}\left(y_{0}, \cdots, y_{i}\right)=$

$\ln p_{1}\left(y_{0}, \cdots, y_{i-1}\right)+\ln p_{1}\left(y_{i} \mid y_{0}, \cdots, y_{i-1}\right), \quad 0 \leq i \leq n-1$.

Since the joint distribution of $\left\{Y_{0}, \cdots, Y_{i}\right\}$ is Gaussian, the conditional distribution $p_{1}\left(y_{i} \mid y_{0}, \cdots, y_{i-1}\right)$ is also Gaussian with mean $\mathbb{E}_{1}\left\{Y_{i} \mid y_{0}, \cdots, y_{i-1}\right\}$ and variance denoted $\sigma_{e, i}^{2}$. The log-likelihood up to the $i$ th observation along a route can thus be expressed as

$\ln p_{1}\left(y_{0}, \cdots, y_{i}\right)=\ln p_{1}\left(y_{0}, \cdots, y_{i-1}\right)-\frac{1}{2} \ln \left(2 \pi \sigma_{e, i}^{2}\right)-\frac{1}{2} \frac{\widetilde{y}_{i}^{2}}{\sigma_{e, i}^{2}}$,

where $\widetilde{y}_{i} \triangleq y_{i}-\widehat{y}_{i \mid i-1}$ and $\widehat{y}_{i \mid i-1} \triangleq \mathbb{E}_{1}\left\{Y_{i} \mid y_{0}, \cdots, y_{i-1}\right\}$ is the minimum mean square error (MMSE) prediction of $Y_{i}$ given $\left\{Y_{0}, \ldots, Y_{i-1}\right\}$. Note that $\sigma_{e, i}^{2}=\mathbb{E}_{1}\left\{\widetilde{Y}_{i}^{2}\right\}$ is the
MMSE of the predictor and is not a function of the realization $y_{0}, \cdots, y_{i-1}$. The log-likelihood of the observations under $\mathcal{H}_{1}$ is given by evaluating the recursion (6) explicitly for the $(n-1)$ th term, yielding

$$
\ln p_{1}\left(y_{0}, \cdots, y_{n-1}\right)=-\frac{1}{2} \sum_{i=0}^{n-1} \ln \left(2 \pi \sigma_{e, i}^{2}\right)-\frac{1}{2} \sum_{i=0}^{n-1} \frac{\widetilde{y}_{i}^{2}}{\sigma_{e, i}^{2}} .
$$

Now, we return to the definition of the Chernoff information given in (4) and (5). Substituting the innovations representation (7) and standard product form for $p_{0}\left(y_{0}, \cdots, y_{n-1}\right)$, we get Eq. 8 (next page).

\section{B. The Proposed Link Metric}

We seek a link metric that captures the contribution of a particular link to the overall $P_{\mathcal{E}}$ of a route. To facilitate the design of an implementable routing strategy, we seek a metric that satisfies independence (i.e., that the contribution of a given link be independent of past and future links) and additivity. To arrive at such a representation we now make three approximations, valid for large $n$ and at high SNR, and show that we are led to the capacity of the Gaussian channel, with signal power given by the innovations power of the field at the appropriate sensor, as a suitable link metric.

First, suppose that the number of hops in $\mathcal{R}$ is sufficiently large. Then, $\frac{1}{n} \sum_{i=0}^{n-1} Y_{i}^{2}$ converges almost surely to its mean of $\sigma_{W}^{2}$ under $\mathcal{H}_{0}$ by the Strong Law of Large Numbers (SLLN). Hence, the last term in the argument of the exponential function in (8) can be approximated by $\frac{n}{2}$ which does not depend on the topology of the route. Therefore, for large $n$ we have Eq. 9 (next page).

Next consider the term involving $\widetilde{Y}_{i}$ above. A straightforward argument using Chebyshev's inequality [1] shows that the term $\frac{\widetilde{Y}_{i}^{2}}{\sigma_{e, i}^{2}}$ can be made arbitrarily small, with probability arbitrarily close to 1 , under $\mathcal{H}_{0}$ as the SNR is increased (recall that $\sigma_{e, i}^{2}$ is the variance of $\widetilde{Y}_{i}^{2}$ under $\mathcal{H}_{1}$ ). Therefore, the sum involving $\tilde{Y}_{i}$ in (9) is negligible at high SNR and we have

$$
\mu(s) \approx s\left\{-\sum_{i=0}^{n-1} \frac{1}{2} \ln \sigma_{e, i}^{2}+\frac{n}{2}\left(\ln \sigma_{W}^{2}+1\right)\right\} .
$$

Combining (3) and (10) yields

$$
P_{\mathcal{E}} \leq B_{c} \approx \pi_{0}^{1-s} \pi_{1}^{s} e^{-s\left\{\sum_{i=0}^{n-1}\left(\frac{1}{2} \ln \frac{\sigma_{e, i}^{2}}{\sigma_{W}^{2}}-\frac{1}{2}\right)\right\}}
$$

where $0 \leq s \leq 1$. Note that optimization over the variable $s$ is separable from optimization over the route topology (i.e., maximization of the sum in (11)). Second, since at high SNR 


$$
\mu(s)=\ln \mathbb{E}_{0}\left\{\exp \left[s\left(-\frac{1}{2} \sum_{i=0}^{n-1} \ln \left(2 \pi \sigma_{e, i}^{2}\right)-\frac{1}{2} \sum_{i=0}^{n-1} \frac{\tilde{Y}_{i}^{2}}{\sigma_{e, i}^{2}}+\frac{n}{2} \ln \left(2 \pi \sigma_{W}^{2}\right)+\frac{1}{2} \sum_{i=0}^{n-1} \frac{Y_{i}^{2}}{\sigma_{W}^{2}}\right)\right]\right\}
$$

$$
\mu(s) \approx \ln \mathbb{E}_{0}\left\{\exp \left[s\left(-\frac{1}{2} \sum_{i=0}^{n-1} \ln \left(2 \pi \sigma_{e, i}^{2}\right)-\frac{1}{2} \sum_{i=0}^{n-1} \frac{\widetilde{Y}_{i}^{2}}{\sigma_{e, i}^{2}}+\frac{n}{2} \ln \left(2 \pi \sigma_{W}^{2}\right)+\frac{n}{2}\right)\right]\right\}
$$

$\sigma_{e, i}^{2} / \sigma_{W}^{2} \gg 1$, the Chernoff information (5) is attained at $s=$ 1 , and is approximately given by

$$
C\left(p_{0}, p_{1}\right) \approx \sum_{i=0}^{n-1}\left(\frac{1}{2} \ln \frac{\sigma_{e, i}^{2}}{\sigma_{W}^{2}}-\frac{1}{2}\right) \approx \sum_{i=0}^{n-1} \frac{1}{2} \ln \frac{\sigma_{e, i}^{2}}{\sigma_{W}^{2}}
$$

Therefore, we have reduced (8) to a form in which the contribution of each link to the $P_{\mathcal{E}}$ of a route is explicit. In particular, (12) reveals that the term

$$
\bar{C}_{i} \triangleq \frac{1}{2} \ln \frac{\sigma_{e, i}^{2}}{\sigma_{W}^{2}}
$$

quantifies the contribution of the $i$ th link to the $P_{\mathcal{E}}$ of a route. Next, note that

$$
\begin{aligned}
\sigma_{e, i}^{2} & =\mathbb{E}_{1}\left[\tilde{Y}_{i}^{2}\right]=\mathbb{E}_{1}\left[\left(Y_{i}-\mathbb{E}_{1}\left[Y_{i} \mid Y_{0}, \ldots, Y_{i-1}\right]\right)^{2}\right] \\
& =\mathbb{E}_{1}\left[\left(S_{i}+W_{i}-\mathbb{E}_{1}\left[S_{i} \mid Y_{0}, \ldots, Y_{i-1}\right]\right)^{2}\right]=\sigma_{W}^{2}+P_{i \mid i-1},
\end{aligned}
$$

where $P_{i \mid i-1} \triangleq \mathbb{E}_{1}\left\{S_{i}-\mathbb{E}_{1}\left[S_{i} \mid Y_{0}, \ldots, Y_{i-1}\right]\right\}^{2}$ is interpreted as the power of the signal innovation. Substituting (14) into (13), we get

$$
\bar{C}_{i}=\frac{1}{2} \ln \left(1+\frac{P_{i \mid i-1}}{\sigma_{W}^{2}}\right),
$$

which is our proposed link metric. Note that the link metric $\bar{C}_{i}$ is additive in the sense that the Chernoff information is monotonic in $\sum_{i} \bar{C}_{i}$. The link metric is not, in general, independent from link to link. However, this issue will be addressed further in the next Section.

The link metric defined in (15) has a well recognized form: it is the capacity of a Gaussian channel with average power $P_{i \mid i-1}$. While the connection between the Chernoff information over a route and the capacity on a link is not obvious, there are several intuitions that support the use of a link metric in (15). The Schweppe's recursion (6) already suggests that the accumulation of likelihood should be made by propagating the innovation variable $\widetilde{Y}_{i}$. We can then interpret (15) as the amount of uncertainty resolved by collecting a sample from node $N_{i}$, and the optimal route as the one that provides the maximum reduction in uncertainty.

\section{Cooperative Routing for the Detection of A GaUSS-MARKov Signal Field}

In the remainder of this paper we assume that the correlation is described by the Gauss-Markov model on each route. Focusing on this special case allows us to describe an optimal cooperative data aggregation scheme in a simple manner, and to explicitly evaluate the link metric (15) in terms of the system parameters, which provides insights that would not be available otherwise. Similar derivations are possible under other correlation models (e.g., the general class of autoregressive models), however, they will not be pursued here.

\section{A. The Gauss-Markov Field and State-Space Representation}

We assume that for any open simple route $\mathcal{R}$ contained in the sensor field, the signal along the route forms a onedimensional stationary Gauss-Markov process and the signal dynamics are given by

$$
\frac{d S(l)}{d l}=-A S(l)+B U(l), \quad 0 \leq l \leq|\mathcal{R}|,
$$

where $|\mathcal{R}|$ denotes the length of the route $\mathcal{R}, A \geq 0$ and $B$ are known constants, and where the initial condition is given by $S(0)$ which has Gaussian distribution $\mathcal{N}\left(0, \sigma_{S}^{2}\right)$ with $\sigma_{S}^{2}=$ $\frac{B^{2}}{2 A}$. The process noise $U(l)$ is zero-mean white Gaussian with unit variance which is independent of $S(0)$. The above assumption is a simplified version of the correlation structure that may be encountered in practice, but it is reasonable for a class of curves (e.g.,those approximating straight lines) that are contained in a homogeneous Gauss-Markov random field.

Note that $A$ represents the correlation strength (or "diffusion rate") of the signal field with respect to distance. A larger value of $A$ implies weaker correlation between two spatial signal samples. A direct calculation reveals the correlation matrix of the signal samples along the route $\mathcal{R}\left(N_{0}, \ldots, N_{n-1}\right)$ to be given by

$$
\boldsymbol{\Sigma}_{S}(i, j)=\sigma_{S}^{2} \exp \left\{-A \sum_{k=\min (i, j)}^{\max (i, j)-1} \Delta_{k}\right\} .
$$

Note that $\boldsymbol{\Sigma}_{S}$ is not generally Toeplitz since the spacing between sensors is not generally uniform.

Under the assumed Gauss-Markov model, the dynamics of signal sample $S_{i}$ at node $N_{i}$ is described by the following state-space model:

$$
\begin{aligned}
S_{i+1} & =a_{i} S_{i}+U_{i}, \quad i=0,1, \cdots, n-2, \\
a_{i} & =e^{-A \Delta_{i+1}}, \quad U_{i} \sim \mathcal{N}\left(0, \sigma_{S}^{2}\left(1-a_{i}^{2}\right)\right),
\end{aligned}
$$

where $S_{0} \triangleq S(0)$.

\section{B. Kalman Aggregation}

We now describe an optimal data aggregation algorithm that provides optimal detection performance while requiring a fraction of the energy of sending raw data from all sensors 
TABLE I

$$
\text { KALMAN AGGREGATION }\left(\widehat{y}_{i \mid i-1} \triangleq \mathbb{E}_{1}\left[Y_{i} \mid y_{0}, y_{1}, \cdots, y_{i-1}\right]\right)
$$

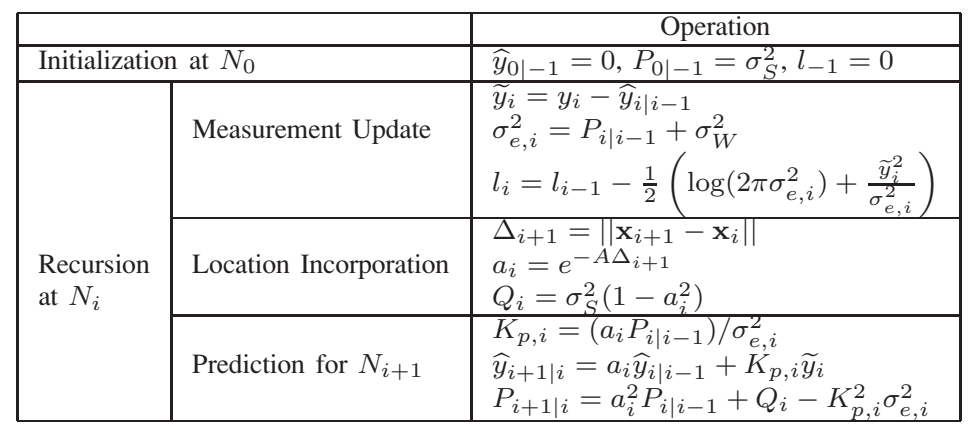

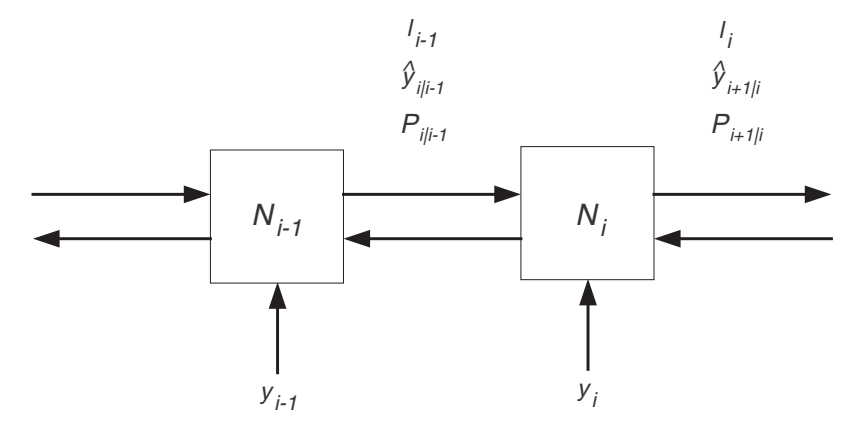

Fig. 3. Information flow along a fusion route.

along the route. A schematic is shown in Fig 3 and the detailed implementation in Table I.

From Schweppe's recursion (6), we see that the loglikelihood up to the $i$ th sensor can be calculated recursively based on information located at sensor $N_{i-1}$ and its own measurement. The key variable that needs to be delivered from $N_{i-1}$ to $N_{i}$ is the predicted observation $\widehat{y}_{i \mid i-1}$ by $N_{i-1}$ based on measurements from all previous sensors in the route. The communication cost for link $i$ has three components, i.e. to obtain the likelihood function at node $i$, three quantities have to arrive at $N_{i}$ from $N_{i-1}$ :

1) The accumulated likelihood function $l_{i-1} \triangleq$ $\ln p_{1}\left(y_{0}, \cdots, y_{i-1}\right)$.

2) The variance of innovation $P_{i \mid i-1}$. This quantity gives the mean-square error of the prediction $\sigma_{e, i}^{2}=P_{i \mid i-1}+$ $\sigma_{W}^{2}$ and specifies the second term of (6).

3) The predicted measurement $\widehat{y}_{i \mid i-1}$. With $\widehat{y}_{i \mid i-1}$ and its own measurement $y_{i}$, prediction error can be generated $\widetilde{y}_{i}=y_{i}-\widehat{y}_{i \mid i-1}$ at sensor $N_{i}$. Along with $\sigma_{e, i}^{2}, \widetilde{y}_{i}$ gives the last term of (6).

If each node stores the relative distance to its nearest neighbor $\left(\Delta_{i}\right)$, this information need not be transmitted.

It is important to point out that the transmission energy used by a particular node in a route is a constant, regardless of the node's position in the route. This is in contrast to the naive noncooperative scheme for which nodes closer to the fusion center deliver successively more measurements to their next neighbor, producing an accumulative ("snow ball") effect in the energy consumed by each sensor along a route. The processing energy at sensor $N_{i}$ is also constant under our cooperative strategy. This can be seen from the operations defined in (6).

\section{Link Metric and Properties}

The Gauss-Markov model enables us to make a key simplification on the link metric (15), connecting the variance of innovation to link length $\Delta_{i}$. From the Kalman recursion, the power in the signal innovation is given by

$$
P_{i \mid i-1}=\frac{\sigma_{W}^{2} a_{i-1}^{2} P_{i-1 \mid i-2}}{P_{i-1 \mid i-2}+\sigma_{W}^{2}}+Q_{i-1},
$$

where $Q_{i-1} \triangleq \sigma_{S}^{2}\left(1-e^{-2 A \Delta_{i}}\right)$. Since $P_{i-1 \mid i-2}$ depends only on $\left\{\Delta_{1}, \cdots, \Delta_{i-1}\right\}$, the Kalman gain $K_{i-1} \triangleq$ $\frac{P_{i-1 \mid i-2}}{P_{i-1 \mid i-2}+\sigma_{W}^{2}}\left(0 \leq K_{i-1} \leq 1\right)$ is a constant with respect to $\Delta_{i}$. Thus, the innovation variance at link $i$ is given by

$$
\begin{aligned}
\sigma_{e, i}^{2} & =P_{i \mid i-1}+\sigma_{W}^{2} \\
& =\sigma_{W}^{2} a_{i-1}^{2} \frac{P_{i-1 \mid i-2}}{P_{i-1 \mid i-2}+\sigma_{W}^{2}}+Q_{i-1}+\sigma_{W}^{2} \\
& =\sigma_{S}^{2}+\sigma_{W}^{2}-\left(\sigma_{S}^{2}-\sigma_{W}^{2} K_{i-1}\right) e^{-2 A \Delta_{i}}
\end{aligned}
$$

where (16) is obtained by substituting $a_{i-1} \triangleq e^{-A \Delta_{i}}$. Thus, for the special case of the Gauss-Markov model, the metric $\bar{C}_{i}$ can be evaluated explicitly, yielding

$$
\begin{array}{r}
\bar{C}_{i}=\frac{1}{2} \ln \left\{\mathrm{SNR}+1-\left(\mathrm{SNR}-K_{i-1}\right) e^{-2 A \Delta_{i}}\right\} \\
1 \leq i \leq n-1,
\end{array}
$$

where the initial value $C_{0}=\frac{1}{2} \ln (1+\mathrm{SNR})$.

As expected, (17) depends on previous links through the Kalman gain. A good approximation that models the link contributions as independent can be obtained when the field is of weak or moderate correlation by substituting $K_{i-1}=1$ in (16). Such an approach will prove useful in an efficient implementation of optimized routing (to be described in Section V). Under this approximation, the link metric becomes

$$
\begin{array}{r}
C_{i} \triangleq \frac{1}{2} \ln \left\{\mathrm{SNR}+1-(\mathrm{SNR}-1) e^{-2 A \Delta_{i}}\right\}, \\
\text { for } 1 \leq i \leq n-1,
\end{array}
$$

which is our proposed link metric for the special case of the Gauss-Markov model.

By expressing the link metric $C_{i}$ as a function of link length, we are able to investigate the effect of sensor spacing on detection performance. We now present properties of $C_{i}$ as a function of $\Delta_{i}$. 
Theorem 1: For SNR $>1$, the link metric $C_{i}$ has the following properties:

(i) $C_{i}$ is a strictly increasing function of $\Delta_{i}$,

(ii) $C_{i}$ converges to $\frac{1}{2} \ln (1+\mathrm{SNR})$ exponentially fast as $\Delta_{i} \rightarrow \infty$, and

(iii) $C_{i}$ is a strictly concave function of $\Delta_{i}$.

Proof: From (17) the convergence to $\frac{1}{2} \log (1+\mathrm{SNR})$ regardless of the value of $K_{i-1}$ and its rate are straightforward since $\lim _{x \rightarrow \infty} \frac{\log c_{1}-\log \left(c_{1}-c_{2} e^{-x}\right)}{e^{-x}}=c_{2} / c_{1}$ for $c_{1}>c_{2}>0$. The partial derivative of $C_{i}$ with respect to $\Delta_{i}$ is given by

$$
\frac{\partial C_{i}}{\partial \Delta_{i}}=\frac{A\left(\mathrm{SNR}-K_{i-1}\right) e^{-2 A \Delta_{i}}}{\mathrm{SNR}+1-\left(\mathrm{SNR}-K_{i-1}\right) e^{-2 A \Delta_{i}}} .
$$

Since $0 \leq K_{i-1} \leq 1$, SNR $>1$ implies $\frac{\partial C_{i}}{\partial \Delta_{i}}>0$ and the link metric is a strict increasing function of $\Delta_{i}$. The second partial derivative is given by

$$
\frac{\partial^{2} C_{i}}{\partial \Delta_{i}^{2}}=\frac{-2 A^{2}(\mathrm{SNR}+1)\left(\mathrm{SNR}-K_{i-1}\right) e^{-2 A \Delta_{i}}}{\left(\mathrm{SNR}+1-\left(\mathrm{SNR}-K_{i-1}\right) e^{-2 A \Delta_{i}}\right)^{2}} .
$$

Therefore, for SNR $>1, \frac{\partial^{2} C_{i}}{\partial^{2} \Delta_{i}}<0$ and the metric is a strictly concave function of $\Delta_{i}$.

Remark 1: The maximal information that a link can provide is given by

$$
C_{\max } \triangleq \lim _{\Delta_{i} \rightarrow \infty} C_{i}=\frac{1}{2} \ln (1+\mathrm{SNR}),
$$

which is achieved in the limit as $\Delta_{i} \rightarrow \infty$ (i.e., when the sensors make independent observations). Even with a finite $\Delta_{i}$, we can achieve most of $C_{\max }$ due to the exponential convergence.

Figure 4(a) shows $C_{i}$ as a function of the link length $\Delta_{i}$. It is seen that the value of link metric increases with $\Delta_{i}$ and eventually converges. This is because as the distance between two sensors increases, their observations approach independence; further increases in $\Delta_{i}$ are of diminishing benefit.

When $\Delta_{i} \rightarrow 0$, the information for the link converges to $\frac{1}{2} \ln 2>0$. As expected, the link information is positive even as the inter-node spacing diminishes to zero. This is because the additive noise is assumed independent from sensor to sensor. However, we observe that the limiting value is independent of $\sigma_{W}^{2}$. This unintuitive result is likely due to the series of approximations necessary to arrive at (18). As another informative metric, we also study the link efficiency, defined as the information per unit energy $C_{i} / E_{i}$. Suppose that we place two sensors at distinct positions that are arbitrarily close. We might expect the link efficiency of this scheme to be large since no transmission energy is required $\left(E_{T}=0\right)$. However, this is not the case as long as the processing energy $E_{P}$ is strictly positive. Figure 4(b) shows the link efficiency, $\frac{C_{i}}{E_{i}}$, as a function of the link length $\Delta_{i}$ for several values of $E_{P}$ when $A=1$, SNR $=10 \mathrm{~dB}, \nu=2$, and $E_{T}=1$. It is seen that the information per unit energy is not maximized as $\Delta_{i} \rightarrow 0$. Rather, $\frac{C_{i}}{E_{i}}$ initially increases as $\Delta_{i}$ increases, reaches a maximum, and then decays to zero as $\Delta_{i}$ further increases.

\section{Shortest Path Routing}

In this section we propose an efficient implementation of Chernoff information-based routing (henceforth, referred to as Chernoff routing) using the shortest path framework. It is shown that our cooperative approach leads to better detection performance for the same network energy consumption. The performance figures included in the section have been reproduced from [45].

In shortest path routing [3], each link in the network is assigned a link cost which quantifies the system resources that are consumed when that link is used (relevant examples are given below). Routes are determined as follows: suppose that a detection process is initiated at node $N_{0}$. Then $N_{0}$ routes its information to the fusion center using the "least cost" route, where the cost of a route is simply the sum of its link costs. Let $\gamma_{i, j}$ be the cost assigned to link the from sensor $i$ to sensor $j$ for all $i$ and $j$ in the network, and set $\gamma_{i, j}=\infty$ if no direct link exists between nodes $i$ and $j$ (due to limited communications range). Some examples of routing strategies and corresponding link costs are (below, we consider $(i, j)$ node pairs that are capable of direct communications, i.e,. $\left.\gamma_{i, j}<\infty\right)$ :

1. Minimum-Hops routing. Each link is assigned the same cost. Thus

$$
\gamma_{i, j}=\epsilon,
$$

for all $i, j$ and for some $\epsilon>0$. This strategy routes data along the path which requires the least number of link traversals from source to the fusion center.

2. Minimum-Energy routing. Each link is assigned the energy value that would be required to transmit from sensor $i$ to sensor $j$, i.e.,

$$
\gamma_{i, j}=E_{i, j}
$$

where $E_{i, j}$ and $\Delta_{i, j}$ are, respectively, the necessary transmission energy (see Section II-B) and the Euclidean distance between arbitrary sensors $i$ and $j$ in the network. This strategy routes information using the path which requires the least total transmission energy from source to the fusion center.

3. Chernoff routing. We now propose a link cost assignment for Chernoff routing based on (18). We cannot apply (18) directly as our link metric since this would result in minimizing the accumulated sum $\sum_{i} C_{i}$ of each route; the opposite of what we desire. Similarly, we cannot simply take $\gamma_{i, j}$ to be a monotonically decreasing function of the link metric (e.g., the negative of (18)), since this strategy does not converge in the absence of a stopping constraint. Thus, we use a metric which seeks to maximize the negative of (18), but with an energy constraint. Specifically, let

$$
\gamma_{i, j}=\left(E_{i, j}-\lambda C_{i, j}\right)_{\epsilon}^{+},
$$

where $C_{i, j}$ is the link metric (18) between nodes $i$ and $j$, where $\lambda \geq 0$ is a weighting factor between the detection performance and energy constraint, and where

$$
(x)_{\epsilon}^{+}=\left\{\begin{array}{lll}
x & \text { if } & x>0 \\
\epsilon & \text { if } & x \leq 0 .
\end{array}\right.
$$

Note that minimum-energy routing corresponds to the case that $\lambda=0$, while min-hops routing corresponds to 


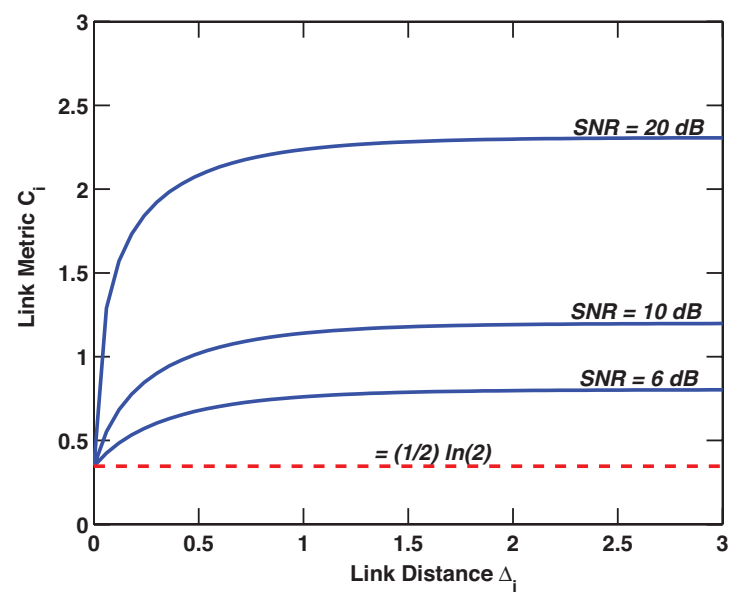

(a)

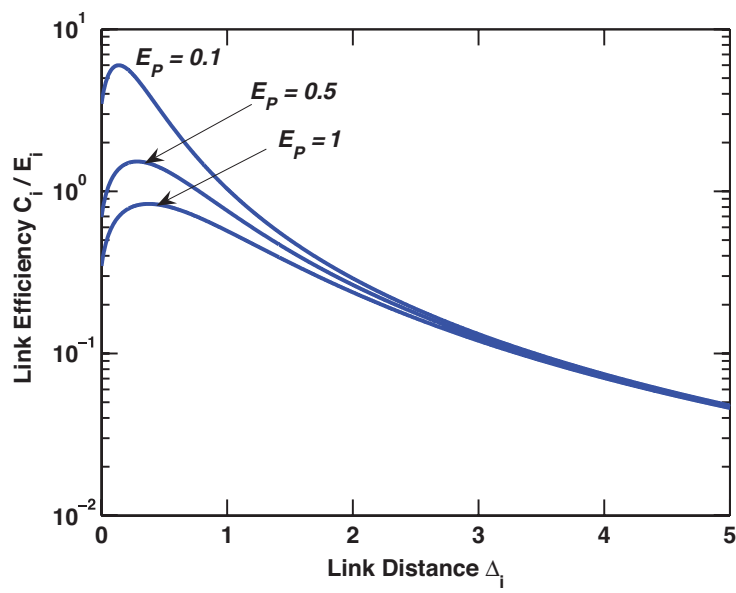

(b)

Fig. 4. The link metric $C_{i}$ as a function of link length $\Delta_{i}$ : (a) $C_{i}$ versus $\Delta_{i}$ for $A=1$, (b) Link efficiency, $\frac{C_{i}}{E_{i}}$, versus $\Delta_{i}$ for $A=1, \mathrm{SNR}=10 \mathrm{~dB}$, $\nu=2, E_{T}=1$, and several values of $E_{P}$.

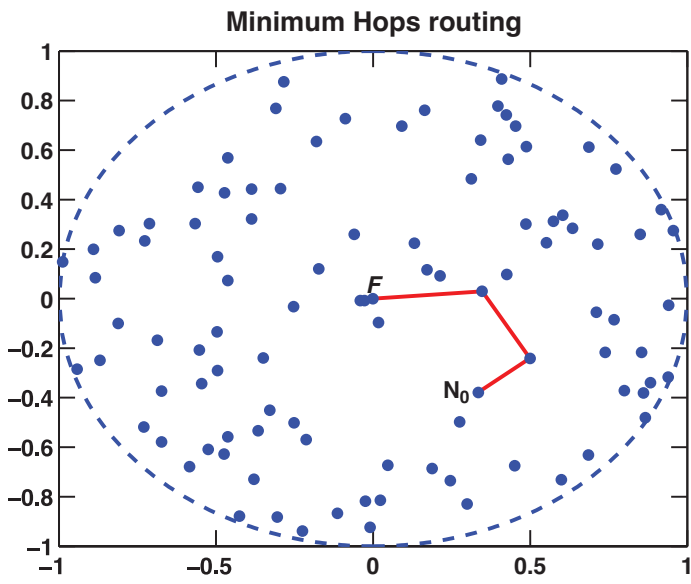

(a)

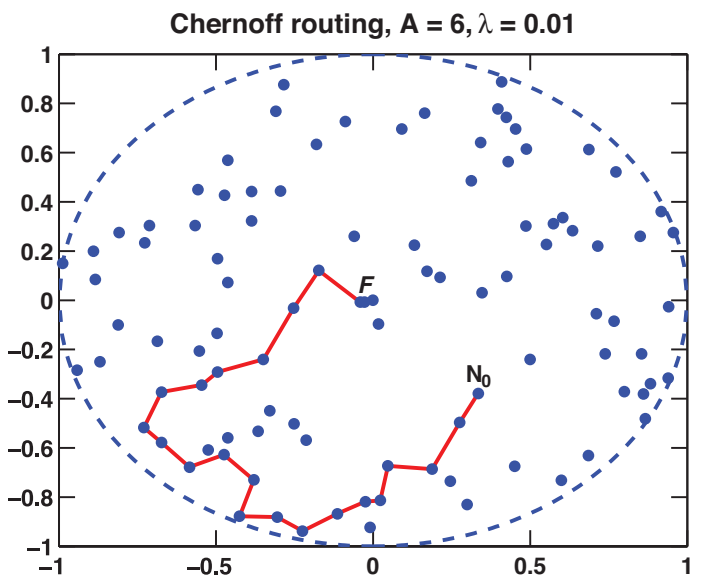

(c)

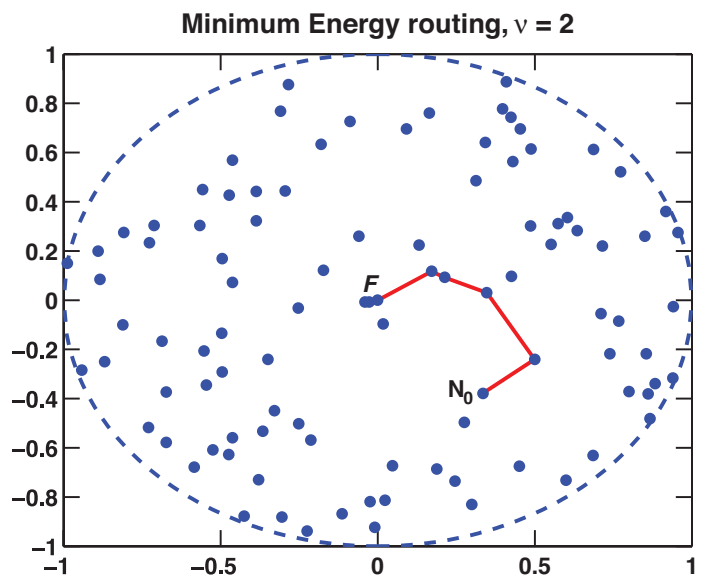

(b)

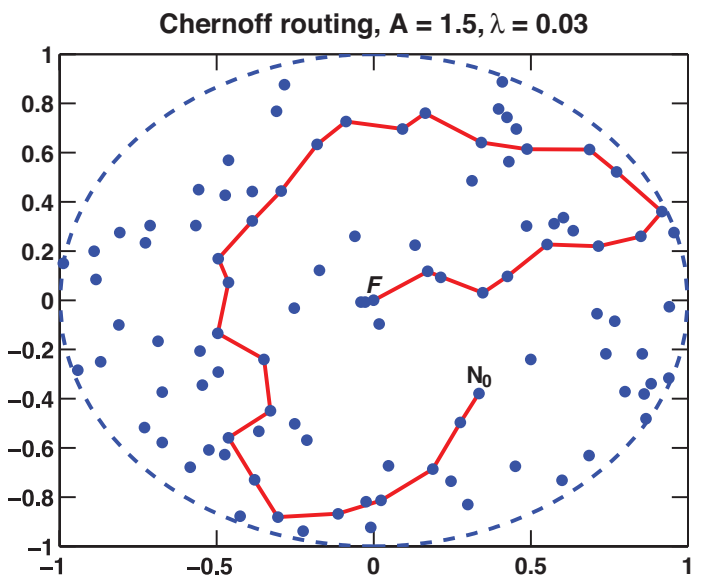

(d)

Fig. 5. The shortest path route from node $N_{0}$ to the fusion center $F$ under each three different routing strategies: (a) Minimum-Hops routing, (b) MinimumEnergy routing, (c) Chernoff routing when $A=6$ and $\lambda=0.01$, and (d) Chernoff routing when $A=1.5$ and $\lambda=0.03$. Common parameters: SNR $=15$ $\mathrm{dB}, \nu=2, \mathcal{N}=100, E_{T}=1, E_{P}=0$, and $\pi_{0}=0.75$.

the case $\lambda \rightarrow \infty$. Although not required, $\epsilon>0$ could be chosen to be an extremely small number in practice to ensure the smooth behavior of the routing assignments with respect to $\lambda$. 


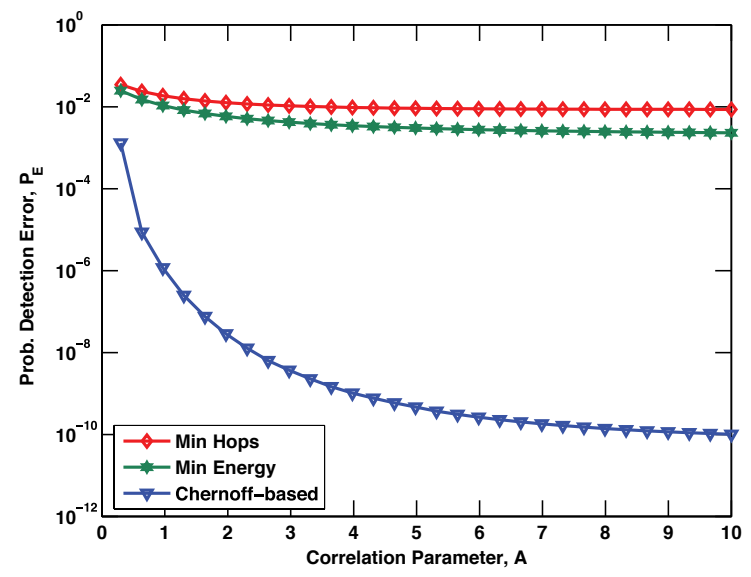

Fig. 6. The probability of detection error $P_{\mathcal{E}}$ for each of the routing schemes for the topology and parameters given in Figure 5. Here, $A$ is variable $(x-$ axis) and $\lambda$ is optimized at each value of $A$.

\section{A. Local Analysis}

We now evaluate the performance of Chernoff routing relative to the two conventional schemes described above. In all simulations in this paper, we consider a circular network of radius one. The locations of $\mathcal{N}$ sensors are then independently and uniformly generated on the disk. Sensors with a Euclidean distance of 0.4 are assumed to be capable of direct communications, and the fusion center is always located at the center of the disk.

We start by comparing the performance of each of the routing strategies (19), (20), (21) for a fixed topology. Figure 5 shows one realization of the network topology and the optimal route from a node $N_{0}$ to the fusion center $F$ for the three routing schemes when $\mathcal{N}=100$ and $\mathrm{SNR}=15$ $\mathrm{dB}$ (other parameters are given in the caption). Note that minimum-hops routing takes a few, long links to the fusion center, whereas minimum-energy routing attempts to break the route into as many small links as possible. On the other hand, Chernoff routing traverses a large region of the network before eventually arriving at the fusion center. Note that when the field is more correlated (Figure 5(d)), Chernoff routing traverses more links before collecting sufficient information and arriving at the fusion center. In the cases of Chernoff routing, $\lambda$ was optimized for the given channel conditions. Note that Chernoff routing requires knowledge of the SNR and correlation parameter $A$ whereas the two conventional routing schemes do not.

In Figure 6, we plot the overall probability of detection error $P_{\mathcal{E}}$ for each of routing schemes when the detection process originates at node $N_{0}$. As a realistic model for the true signal field correlation, we assume that the actual correlation between two sensors is a function of their Euclidean distance as summarized in the following assumption:

Assumption 1: The correlation between sensors $N_{i}$ and $N_{j}$ in the route $\mathcal{R}$ is given by

$$
\boldsymbol{\Sigma}_{S}(i, j)=\sigma_{S}^{2} \exp \left\{-A\left\|\mathbf{x}_{i}-\mathbf{x}_{j}\right\|\right\} .
$$

That is, while the Chernoff routing scheme is established under the Gauss-Markov model, the error probability $P_{\mathcal{E}}$ is determined assuming that the actual signal correlation between any two nodes $i$ and $j$ is given by the more realistic model of Assumption 1. For each value of $A$ we have optimized over $\lambda$ numerically. When $A=1$ Chernoff routing provides approximately four orders of magnitude improvement in $P_{\mathcal{E}}$ compared to the other routing strategies. As expected, Chernoff routing results in a significantly lower error floor in the limit of i.i.d. sensor observations $(A \gg 1)$ compared to the other routing strategies. This is because, for i.i.d. observations, $P_{\mathcal{E}}$ is determined only by the number of sensor observations before arriving at the fusion center, a metric for which Chernoff routing dominates. What is not so clear at this stage is if Chernoff routing remains the best-performing strategy when performance is normalized by the amount of energy consumed (clearly, Chernoff routing consumes more energy than the other strategies; see previous topology figures). However it will be seen in the next subsection that Chernoff routing also maximizes the detection efficiency .

The proceeding analysis was for a fixed node. Next, we consider performance averaged over the network topology. In Figure 7, we plot the shortest path route from each node to the fusion center for each of the routing strategies. Clearly, minimum-hops routing results in a few, large, welldirected hops to the fusion center, whereas minimum-energy and Chernoff routing take smaller, scattered steps. The nodes which lead to major topological differences between the latter strategies are circled in the figure. Figure $7(d)$ is a plot of the $P_{\mathcal{E}}$ when $\mathcal{N}=100, \mathrm{SNR}=15 \mathrm{~dB}, \pi_{0}=0.75, \lambda=0.01$ for all $A$, and when the true signal correlation is given by Assumption 1. Note that Chernoff routing provides about a 40-percent reduction in the $P_{\mathcal{E}}$ compared to minimum-energy routing.

\section{B. Global Analysis}

We now study global performance, i.e., detection performance averaged over a large number of random network topologies of fixed size. In Figure 8 we provide a comprehensive analysis of the routing characteristics when SNR = $15 \mathrm{~dB}$ and $A=0.5$ (system parameters are given in the figure caption). For each value of network size, we have averaged over realizations of the network topology. Thus, each of the curves in Figure 8 represents the fundamental performance level of the network for a fixed value of the SNR and correlation parameter $A$ as network size $\mathcal{N}$ is varied. Figure 8(a) plots the average number of hops from all potential source nodes to the fusion center. As expected, Minimum-hops routing requires the fewest hops. In Figure 8(b) it is seen that Chernoff routing accumulates the most Chernoff information from all potential source nodes to the fusion center, which is also expected. In Figure 8(c) we plot the average energy consumed by each scheme. Note that Chernoff routing consumes virtually the same amount of energy as Minimum-Energy routing; this is a significant fact since Chernoff routing will be shown to be provide better $P_{\mathcal{E}}$ and Detection Efficiency compared to minimum-energy routing next. In Figure $8(\mathrm{~d})$ we plot the Chernoff efficiency (i.e., a measure of detection performance when normalized by the energy consumed by a particular routing scheme). Note that Chernoff routing provides the best "detection efficiency" 


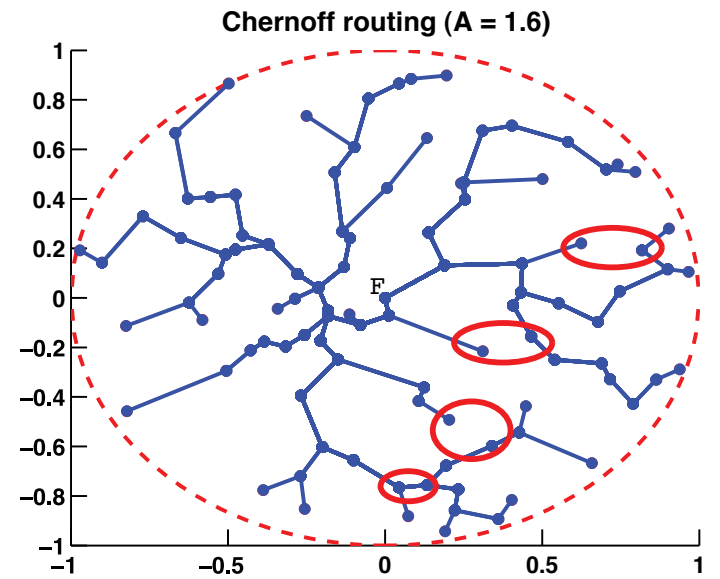

(a)

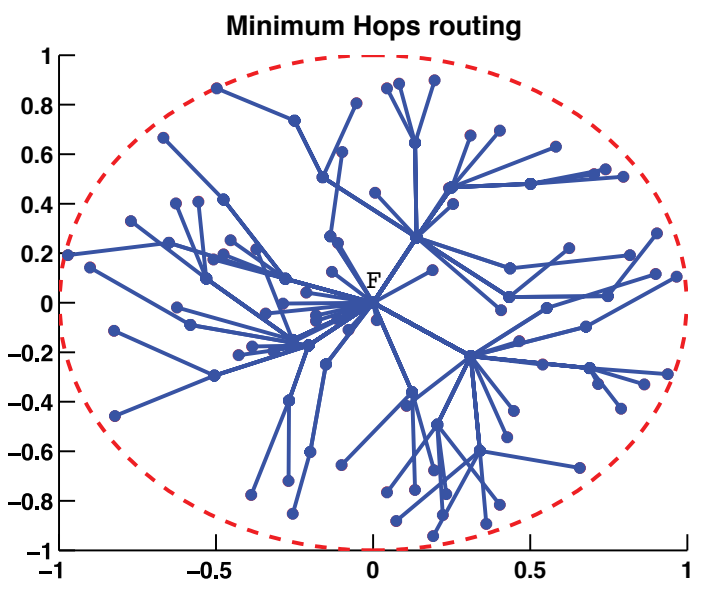

(c)

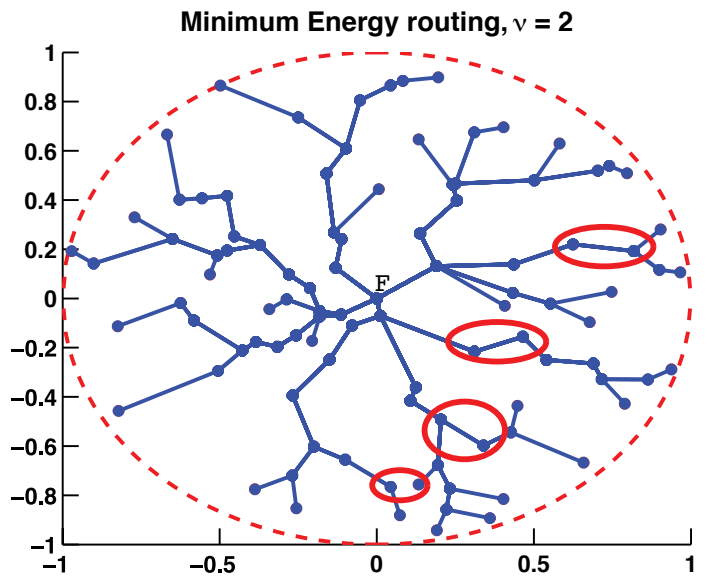

(b)

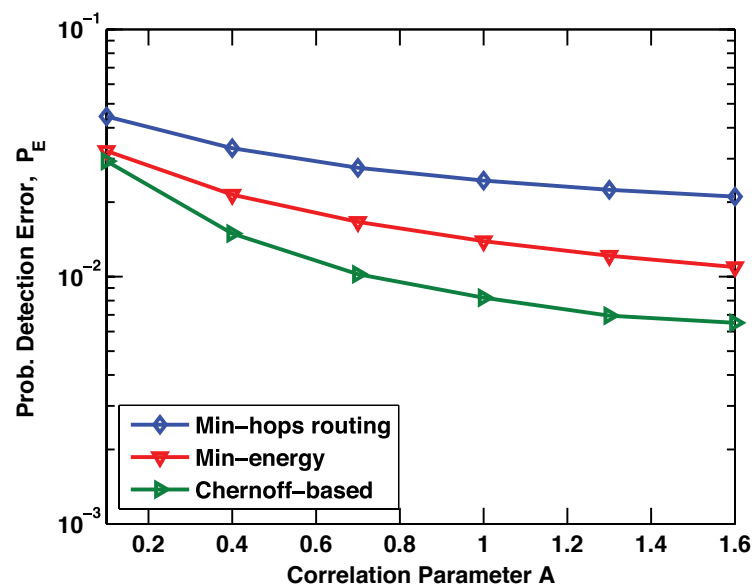

(d)

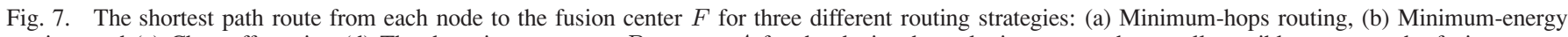

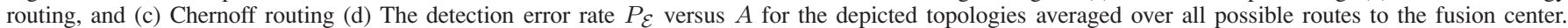
Parameters: $A=1.6, \lambda=0.01, \mathrm{SNR}=15 \mathrm{~dB}, \nu=2, N=100, E_{T}=1$, and $E_{P}=0$.

across the entire range of network sizes tested. We plot the probability of detection error $P_{\mathcal{E}}$ averaged over all potential source nodes in Figure 8(e). Note that Chernoff routing results in better error performance than either of the other routing strategies.

Finally, we consider the energy efficiency of the cooperative data aggregation scheme of Section IV-B. In Figure 8(f), we plot the ratio of the energy used by the noncooperative routing strategy, in which energy consumption increases in the order $O\left(n^{2}\right)$, to that used by cooperative routing, in which energy consumption increases in the order $O(n)$. Note that the curves in Figure 8(f) mimic the average number of hops curves depicted in $8(\mathrm{a})$. This is to be expected. As the average number of hops in the network size increases, the "energy penalty ratio", as quantified above, increases, and noncooperative routing performs increasingly poorly relative to cooperative routing. It is seen that cooperative routing provides large energy savings as the network increases, e.g., a savings by a factor of five for Chernoff based routing when $\mathcal{N}=100$.

\section{CONCLUding REMARKS}

Cooperation means, literally, acting towards a common goal. Such a notion is particularly relevant for sensor networks that are designed not for individual nodes but for a common set of objectives. This paper is an attempt to put classical distributed detection in a multi-hop network setting, addressing directly the detection of conditionally correlated phenomena through route selection with energy constraints. We have presented an approach to cooperative routing for distributed detection in large sensor networks. From a network protocol perspective, the contribution of this paper lies in the definition of a new link metric that characterizes the overall network performance.

Cooperative routing for sensor networks covers a much broader range of issues, of course, and we have left many issues open for future investigation. Note that applying the Chernoff information without a stopping constraint would have made the shortest path algorithm nonconvergent, since there is a tendency to go back and forth in the network while forming a route. By including an energy constraint, we stabilized the routing algorithm so that it yielded sensible routes. But there is still a need to refine our metric and its use. Medium access control has also been ignored completely. 


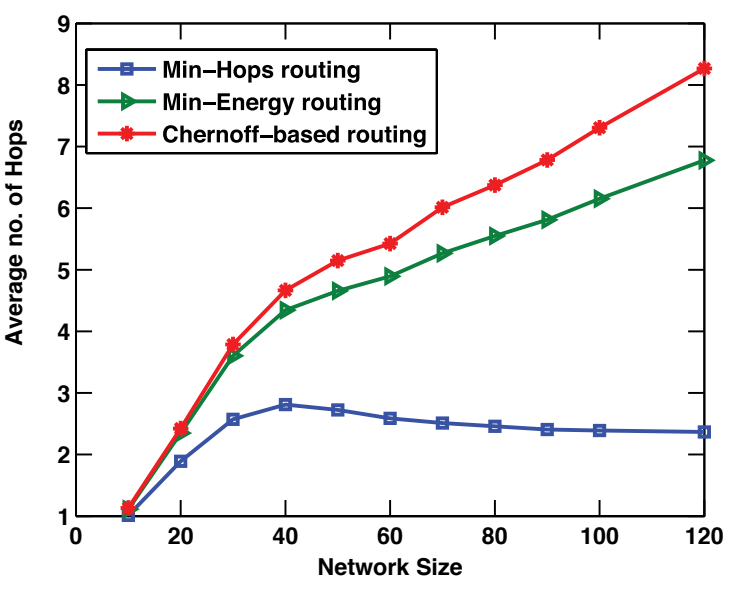

(a)

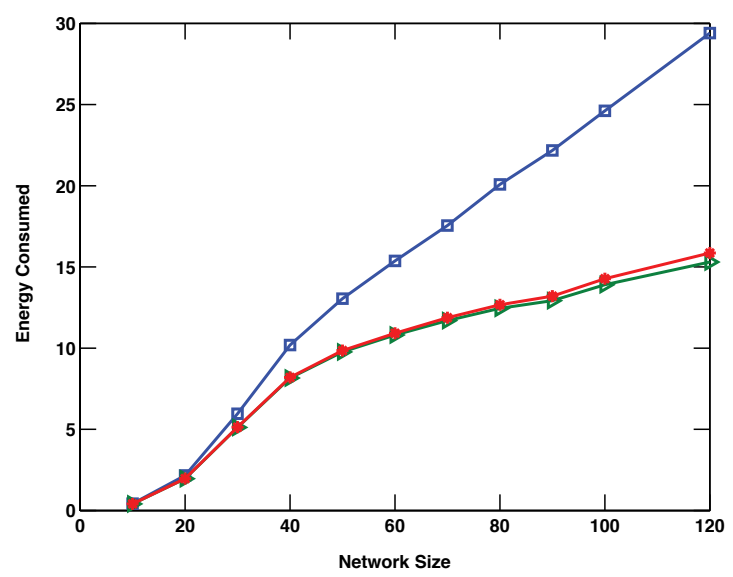

(c)

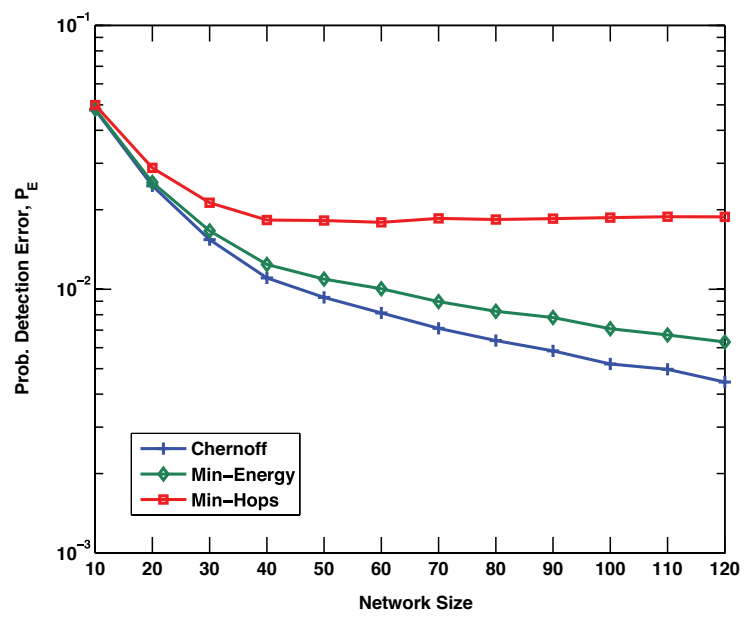

(e)

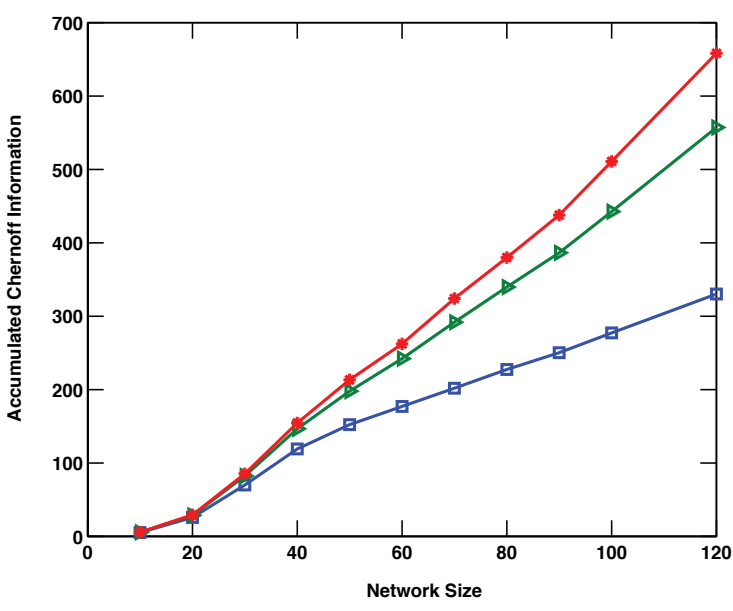

(b)

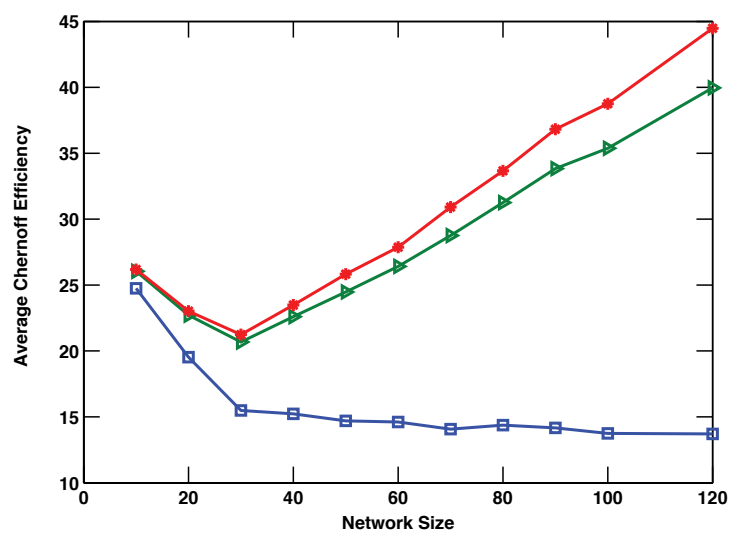

(d)

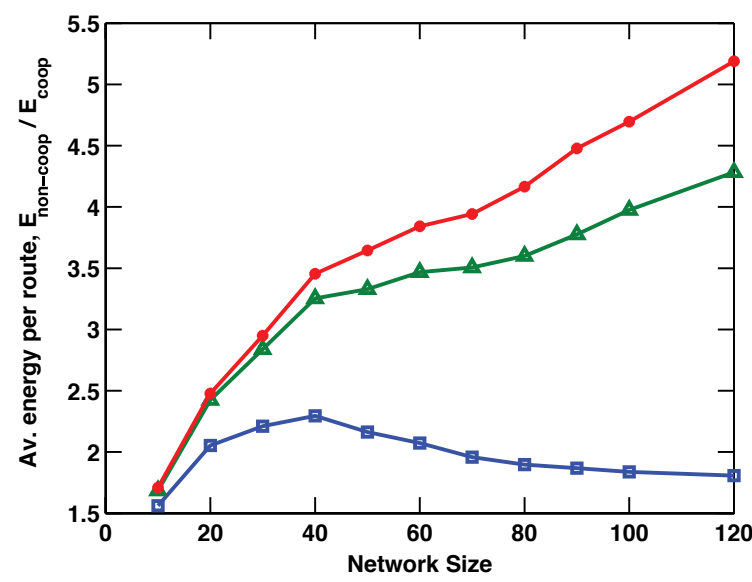

(f)

Fig. 8. Comprehensive performance analysis of the three routing schemes when $\mathrm{SNR}=15 \mathrm{~dB}, A=0.5, \nu=2, \pi_{0}=0.75$, and $\lambda=0.01$ for three different routing strategies: (a) Average no. of hops, (b) Accumulated Chernoff information, (c) Total energy consumed, and (d) Average Chernoff efficiency, (e) Probability of detection error $P_{\mathcal{E}}$, and (f) Energy consumption of cooperative versus noncooperative routing.

While this is not unusual in designing routing strategies, separating medium access control from routing may lead to inefficiency. The problem of local quantization is excluded in the paper. The difficulty here is obtaining an tractable model on which a proper link metric can be derived.

We have not explicitly quantified the overhead cost needed to establish and maintain shortest path routes in the application portion of this paper, Section V. The networks that we 
consider are ad-hoc, but are assumed to have a fairly static topology once deployed. Hence, this overhead can be roughly approximated as a "one-time" deployment cost that is of diminishing influence as the lifetime of the network increases. However, if the network topology is rapidly time varying, then constant route maintenance is required and shortest path routing may not be a reasonable routing strategy. In this case, other approaches to implementation may be needed. Of course, the theoretical results of Section III would remain valid and could potentially prove useful in deriving such an alternate implementation.

We believe that the main contribution of this paper is the mapping of the detection probability to a link metric (even if imperfect), and that this represents a major advance in the distributed detection literature in which correlated sources must route their data to a central authority (such as a fusion center). For contrast, consider the case of circuit switched networks, for which the lack of an effective mapping of blocking probability to a link metric has resulted in a certain level of chaos in the routing algorithms. Routing without a link metric is a combinatorial optimization problem, whereas the existence of link metric results in a shortest path implementation that can be solved efficiently.

\section{REFERENCES}

[1] A. Papoulis, Probability, Random Variables, and Stochastic Processes. McGraw-Hill, 1984.

[2] F. C. Schweppe, "Evaluation of likelihood functions for Gaussian signals," IEEE Trans. on Information Theory, vol. IT-1, pp. 61-70, 1965.

[3] D. Bertsekas and R. Gallager, Data Networks, 2nd Edition, Prentice Hall, New York, 1991.

[4] P. K. Varshney, Distributed Detection and Data Fusion. New York, NY: Springer, 1997.

[5] R. Viswanathan and P.K.Varsheny, "Distributed Detection with Multiple Sensors: Part II -Advanced Topics ," Proceedings of the IEEE, vol. 85, pp. 54-63, Jan. 1997.

[6] R.S.Blum, S.A.Kassam, and H.V.Poor, "Distributed Detection with Multiple Sensors: Part II-Advanced Topics," Proceedings of the IEEE, vol. 85, pp. 64-79, Jan. 1997.

[7] B. Chen, R. Jiang, T. Kasetkasem, and P. Varshney, "Channel aware decision fusion in wireless sensor networks," IEEE Trans. on Signal Processing, vol. 52, pp. 3454-3458, Dec. 2004.

[8] B. Chen and P.Willett, "On the Optimality of the Likelihood-Ratio Test for Local Sensor Decision Rules in the Presence of Nonideal Channels," IEEE Trans. on Information Theory, vol. 51, pp. 693-700, Feb 2005.

[9] G. Mergen and L. Tong, "Estimation Over deterministic multiaccess channels," in Proceedings of the 42nd Allerton Conf. on Communications, Control, and Computing, Monticello, IL, Sep.28-Oct.01 2003.

[10] R.S. Blum and S.A. Kassam, "On the Asymptotic Relative Efficiency of Distributed Detection Schemes," IEEE Transactions on Information Theory, vol. 41, pp. 523-527, March 1995.

[11] K. Liu and A. M. Sayed, "Optimal distributed detection strategies for wireless sensor networks,", in 42nd Annual Allerton Conf. on Commun., Control and Comp., Oct. 2004.

[12] G. Mergen, V. Naware, and L. Tong, "Asymptotic detection performance of type-based multiple access in sensor networks," in Proc. of SPAWC'05, (New York, NY), June 2005.

[13] G. Mergen, V. Naware, and L. Tong, "Asymptotic Detection Performance of Type-Based Multiple Access Over Multiaccess Fading Channels." submitted to IEEE Trans. on Signal Processing, May 2005.

[14] J.-F. Chamberland and V. V. Veeravalli, "Asymptotic results for decentralized detection in power constrained wireless sensor networks," IEEE JSAC Special Issue on Wireless Sensor Networks, 2004.

[15] J.-F. Chamberland and V. V. Veeravalli, "Decentralized Detection in Sensor Networks," IEEE Trans. Signal Processing, vol. 51, pp. 407416, February 2003.

[16] J.-F. Chamberland and V. V. Veeravalli, "Design of sensor networks for detection applications via large-deviation theory," in Proc. of 2004 IEEE Information Theory Workshop, (San Antonio, TX), Oct. 2004.
[17] Y. Sung, L. Tong, and H. Poor, "Neyman-Pearson Detection of GaussMarkov Signals in Noise: Closed-Form Error Exponent and Properties," to appear in IEEE Trans. Information Theory, April 2006.

[18] Y. Sung, S. Misra, L. Tong, and A. Ephremides, "Signal Processing for Application-Specific Ad Hoc Networks," IEEE Signal Processing Magazine, vol. 23, no. 5, pp. 74-83, September 2006.

[19] C. Perkins and P. Bhagwat, "Highly Dynamic Destination-Sequenced Distance-Vector Routing (DSDV) for Mobile Computers," in Proc. of the ACM SIGCOMM, pp. 1-11, 1994.

[20] D. B. Johnson and D. A. Maltz, "Dynamic source routing in ad hoc wireless networks," Mobile Computing, edited by Tomasz Imielinski and Henry F. Korth, pp. 153-181, Kluwer Academic Publishers,, pp. 153181, March 1996.

[21] V. D. Park and S. Corson, "A highly adaptive distributed routing algorithm for mobile wireless networks," in Proc. of IEEE INFOCOM' 97, vol. 3, pp. 1405-1413, 1997.

[22] C. Intanagonwiwat, R. Govindan, and D. Esterin, "Directed Diffusion : A Scalable and Robust Paradigm for Sensor Networks," in Proc. 6th ACM/Mobicom Conference, (Boston,MA), pp. pp 56-67, 2000.

[23] D. Ganesan, R. Govindan, S. Shenker, and D. Estrin, "Highly resilient, energy efficient multipath routing in wireless sensor networks," in Mobile Computing and Communications Review (MC2R), vol. 1, 2002.

[24] N. Sadagopan and A. H. B. Krishnamachari, "The acquire mechanism for efficient querying in sensor networks," in International Workshop on Sensor Network Protocol and Applications, (Anchorage, AK), May 2003.

[25] D. Braginsky and D. Estrin, "Rumor Routing Algorithm for Sensor Networks," in Proc. of the 1st Workshop on Sensor Networks and Applications, (Atlanta, GA), Sept. 2002.

[26] J. Wiesselthier, G. Nguyen, and A. Ephremides, "Energy-aware wireless networking with directional antennas: The case of session-based broadcasting and multicasting," IEEE Trans. Mob. Comput., vol. 1, no. 3, pp. $176-191,2002$

[27] T. ElBatt and A. Ephremides, "Joint scheduling and power control for wireless ad-hoc networks," in INFOCOM, vol. 2, pp. 976 -984, 2002.

[28] T. Girici and A. Ephremides, "Joint routing and scheduling metrics for ad hoc wireless networks," in Proc. of the 36th Asilomar Conference on Signals, Systems and Computers, 2002, pp. 1155 - 1159, Nov. 2002.

[29] L. Yu and A. Ephremides, "Detection performance and energy-efficiency trade-off in a sensor network ", in Proc. of 2003 Allerton Conference, (Allerton, IL), Oct. 2003.

[30] S. Pattem, B. Krishnamachari, and R. Govindan, "The impact of spatial correlation on routing with compression in wireless sensor networks," in Proc. ACM/IEEE International Symposium on Information Processing in Sensor Networks (IPSN), (Berkeley, CA), April 2004.

[31] T. Girici and A. Ephremides, "Optimal power control for wireless queueing networks," in CISS 2004, (Princeton, NJ), March 2004.

[32] H. Chang and L. Tassiulas, "Energy conserving routing in wireless adhoc networks," in Proc. of IEEE INFOCOM 2000, (Tel-Aviv, Israel), pp. 22-31, March 2000.

[33] S. Singh, M. Woo, and C. Raghavendra, "Power-aware routing in mobile ad hoc networks," in Proc. 4th ACM/IEEE Conference on Mobile Computing and Networking (MobiCom), October 1998.

[34] R. Shah and J. Rabaey, "Energy aware routing for low energy ad hoc sensor networks," in Proc. of the 1st Intl. Workshop on Sensor Network Protocol and Applications, May 2003.

[35] W. Heinzelman, J. Kulik, and H. Balakrishnan, "Adaptive Protocols for Information Dissemination in Wireless Sensor Networks," in Proc. 5th ACM/IEEE Mobicom Conference, (Seattle,WA), August 1999.

[36] S. Madden, M. J. Franklin, J. M. Hellerstein, and W. Hong, "TAG: a Tiny AGgregation Service for Ad-Hoc Sensor Networks," in 5th Annual Symposium on Operating Systems Design and Implementation (OSDI), Dec. 2002.

[37] J. Zhao, R. Govindan, and D. Estrin, "Residual energy scans for monitoring wireless sensor networks," in Proc. of IEEE Wireless Communications and Networking Conference (WCNC'02), (Orlando, FL, USA), March 2002.

[38] S.Lindsey and C.S. Raghavendra, "PEGASIS: Power Efficient GAthering in Sensor Information Systems," in Proceedings of the 2002 IEEE Aerospace Conference, Big Sky, MT, March 2002.

[39] B. Krishanamachari, D. Estrin, and S. Wicker, "The impact of data aggregation in wireless sensor networks," in International Workshop on Distributed Event-Based Systems, (DEBS '02), (Vienna, Austria), July 2002.

[40] I. Solis and K. Obraczka, "The Impact of Timing in Data Aggregation for Sensor Networks," in Proc. of ICC 2004, (Paris, France), July 2004.

[41] M. Coates, "Distributed particle filtering for sensor networks," in Proc. of IPSN, (Berkeley, CA), April 2004. 
[42] D. Spanos, R. Olfati-Saber, and R. Murray, "Distributed Kalman Filtering in Sensor Networks with Quantifiable Performance," in Proc. of IPSN 2005, (Los Angeles, CA), April 2005.

[43] H. V. Poor, An Introduction to Signal Detection and Estimation. New York: Springer-Verlag, 1994.

[44] A. Dembo and O. Zeitouni, "Large Deviations Techniques and Applications," Springer, 1998

[45] Y. Sung, S. Misra, L. Tong and A. Ephremides, "A New Link Metric for the Detection of Correlated Signal Fields in Multi-Hop Wireless Sensor Networks," Cornell University Technical Report, No. ACSP-TR-01-0601, January 15, 2006.

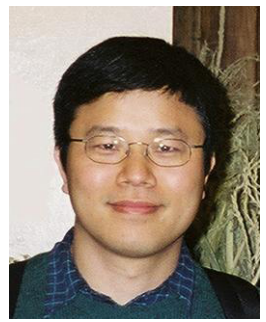

Youngchul Sung received the B.S. and M.S. degrees from Seoul National University, Seoul, Korea in Electronics Engineering in 1993 and 1995, respectively, and Ph.D. degree in Electrical Engineering from Cornell University, Ithaca NY in 2005. Dr. Sung is currently a senior engineer at Qualcomm, Inc. His areas of interest include mobile communication network analysis and design, communication theory, large-scale sensor networks and statistical signal processing.

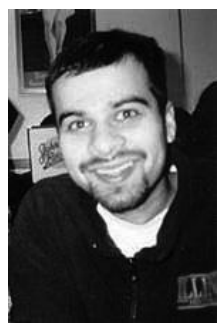

Saswat Misra received the B.S. in electrical engineering from the University of Maryland at College Park in 2000 and the M.S. in electrical engineering from the University of Illinois at Urbana-Champaign in 2002. Since 2002, he has been a Research Scientist at the Army Research Laboratory (ARL) in Adelphi, MD in the Communications and Network Systems division. Mr. Misra has previously worked on optimal training design for wireless communication systems; an area in which he has published several papers and holds two patents (pending). Since 2005, he has been a Ph.D. candidate at Cornell University. He is currently studying routing and security issues in wireless military networks.

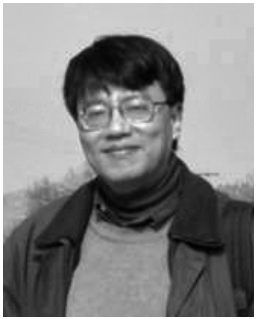

Lang Tong is a Professor in the School of Electrical and Computer Engineering, Cornell University, Ithaca, NY, USA. He received the B.E. degree from Tsinghua University, Beijing, China, in 1985, and M.S. and Ph.D. degrees in electrical engineering in 1987 and 1991, respectively, from the University of Notre Dame, Notre Dame, Indiana. Dr. Tong received the Young Investigator Award from the Office of Naval Research in 1996, the Outstanding Young Author Award from the IEEE Circuits and Systems Society, the 2004 Best Paper Award (with M. Dong) from the IEEE Signal Processing Society, and the 2005 Leonard G. Abraham Prize Paper Award (with P. Venkitasubramaniam and S. Adireddy) from the IEEE Communications Society.

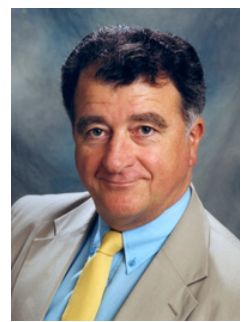

Anthony Ephremides received his B.S. degree from the National Technical University of Athens (1967), and M.S. (1969) and Ph.D. (1971) degrees from Princeton University, all in Electrical Engineering. He has been at the University of Maryland since 1971, and currently holds a joint appointment as Professor in the Electrical Engineering Department and the Institute of Systems Research (ISR). Dr. Ephremides is co-founder of the NASA Center for Commercial Development of Space on Hybrid and Satellite Communications Networks established in 1991 at Maryland as an off-shoot of the ISR. He won the IEEE Donald E. Fink Prize Paper Award (1992). He has been the President of the Information Theory Society of the IEEE (1987), and served on the Board of the IEEE (1989 and 1990). 